\title{
A NOTE ON BERNSTEIN'S APPROXIMATION PROBLEM
}

\section{W. H. J. FUCHS AND HARRY POLLARD}

This note is intended as an addendum to the paper [2] with which we presuppose familiarity.

Vidav [3] has observed that if condition (3) of [2] is satisfied:

(3) there exists a sequence of polynomials $p_{n}$ such that

$$
\lim _{n \rightarrow \infty} p_{n}(u) K(u)=1, \quad\left|p_{n}(u) K(u)\right| \leqq C, \quad-\infty<u<\infty,
$$

then $\left\{u^{n} K(u)\right\}_{0}^{\infty}$ is fundamental in $C_{0}(-\infty, \infty)$, unless $1 / K(z)$ is an entire function.

Unfortunately this criterion is defective in that it leaves in an inconclusive position such functions as $K(u)=e^{-u^{2}}, K(u)=\operatorname{sech} u$ whose reciprocals are entire, which satisfy the condition (3), and yet which are known to give rise to fundamental sets $\left\{u^{n} K(u)\right\}$.

In this note we present a sharper criterion which leaves in this indecisive state a smaller class of functions.

Theorem. If (3) holds, then $\left\{u^{n} K(u)\right\}$ is fundamental in $C_{0}(-\infty$, $\infty)$ unless $1 / K(z)$ is an entire function of exponential type zero.

PROOF. If (3) holds and $\left\{u^{n} K(u)\right\}$ is not fundamental, then necessarily [2]

$$
\int_{-\infty}^{\infty} \frac{\log |K(u)|}{1+u^{2}} d u>-\infty .
$$

Let $F=1 / K$. By virtue of (3) $K$ does not vanish so that $F$ is continuous and

$$
\int_{-\infty}^{\infty} \frac{\log |F(u)|}{1+u^{2}} d u<\infty .
$$

Since $K$ vanishes at $\pm \infty,|F(u)|>1$ for sufficiently large $u$. Therefore the preceding formula is equivalent to

$$
\int_{-\infty}^{\infty} \frac{\log ^{+}|F(u)|}{1+u^{2}} d u<\infty .
$$

According to (3) and the principle of harmonic majorization [1, p. 955]

Received by the editors September 22, 1954 and, in revised form, November 11, 1954. 


$$
\begin{aligned}
\log \left|p_{n}(z)\right| & \leqq \frac{1}{\pi} \int_{-\infty}^{\infty} \frac{|y| \log \left|p_{n}(\xi)\right|}{(x-\xi)^{2}+y^{2}} d \xi \\
& \leqq \frac{1}{\pi} \int_{-\infty}^{\infty} \frac{|y| \log ^{+}|F(\xi)|}{(x-\xi)^{2}+y^{2}} d \xi+\log C \\
& \equiv U(x, y), y \neq 0, z=x+i y .
\end{aligned}
$$

The function $U(x, y)$ is harmonic in $y>0$ and in $y<0$. As $x+i y \rightarrow x_{0}$, $U(x, y) \rightarrow U\left(x_{0}, 0\right)$, by a well-known property of the Poisson integral of a continuous function. Hence $\left|p_{n}(z)\right|$ is majorized by the continuous function $\exp (U(x, y))$. Since $p_{n}(x) \rightarrow 1 / K(x)$ on the real axis, it follows that $\lim _{n \rightarrow \infty} p_{n}(z)$ exists for all $z$ and is an entire function, $F(z)$. On the real axis $F(x)=1 / K(x)$, of course. Letting $n \rightarrow \infty$ in $(B)$ gives

$$
\log |F(z)| \leqq U(x, y) .
$$

In $|y| \geqq \max (|x|, 2)$,

$$
\frac{1+\xi^{2}}{(x-\xi)^{2}+y^{2}}<A .
$$

Also in $\xi^{2}<|z|,|z|>2$

$$
\frac{1+\xi^{2}}{(x-\xi)^{2}+y^{2}}<\frac{B}{|z|} .
$$

Hence in $|y| \geqq \max (|x|, 2)$,

$$
\begin{aligned}
U(x, y)= & \frac{|y|}{\pi} \int_{-\infty}^{\infty} \frac{\log ^{+}|F(\xi)|}{1+\xi^{2}} \frac{1+\xi^{2}}{(x-\xi)^{2}+y^{2}} d \xi \\
= & \frac{|y|}{\pi} \int_{|\xi|^{2}<|z|}+\frac{|y|}{\pi} \int_{\xi^{2} \geqq|z|} \\
< & O\left(\frac{|y|}{|z|} \int_{\xi^{2}<|z|} \frac{\log ^{+}|F(\xi)|}{1+\xi^{2}} d \xi\right) \\
& +O\left(|y| \int_{\xi^{2}>|z|} \frac{\log ^{+}|F(\xi)|}{1+\xi^{2}} d \xi\right)=o(|y|),
\end{aligned}
$$

since the integral in the second $O$-term is $o(1)$, by (A). This proves by (C) that $F(z)$ is of order one, minimum type in $\pi / 4 \leqq|\theta| \leqq 3 \pi / 4$. To complete the proof note that 


$$
\left|p_{n}\left( \pm r e^{ \pm i \pi / 4}\right)\right| \leqq \mathrm{e}^{e r}
$$

We can now apply a standard Phragmén-Lindelöf argument.

The function $f(z)=p_{n}(z) e^{-2^{1 / 2} \text { cs }}$ satisfies

$$
\begin{aligned}
& |f(z)|<K(\epsilon) \\
& |f(z)|<1 \quad\left(|\theta| \leqq \pi / 4,|z|=r_{0}(\epsilon)\right),
\end{aligned}
$$

where $R$ is any sufficiently large positive number. Hence, by the maximum modulus principle

$$
|f(z)|<K(\epsilon)
$$

i.e.

$$
\left|p_{n}(z)\right|<K(\epsilon)\left|e^{2^{1 / 2} \epsilon z}\right|<K(\epsilon) e^{\epsilon|z|}\left(|\theta| \leqq \pi / 4,|z| \geqq r_{0}(\epsilon)\right) .
$$

It follows that there is a function $r_{1}(\eta)$ of $\eta$ such that for every $\eta>0$

$$
\left|p_{n}(z)\right|<e^{\eta|z|} \quad\left(|\theta| \leqq \pi / 4,|z| \geqq r_{1}(\eta)\right) .
$$

Letting $n \rightarrow \infty$ gives

$$
|F(z)| \leqq e^{\eta|z|} \quad\left(|\theta| \leqq \pi / 4,|z| \geqq r_{1}(\eta)\right) .
$$

Similarly this inequality can be proved for the sector $|\pi-\theta| \leqq \pi / 4$, which completes the proof of the theorem.

\section{REFERENCES}

1. L. Carleson, On Bernstein's approximation problem, Proc. Amer. Math. Soc. vol. 2 (1951) pp. 953-961.

2. H. Pollard, Solution of Bernstein's approximation problem, Proc. Amer. Math. Soc. vol. 4 (1953) pp. 869-875.

3. I. Vidav, Sur la solution de H. Pollard du problème d'approximation de S. Bernstein, C. R. Acad. Sci. Paris vol. 258 (1954) pp. 1959-1961.

Cornell University 